\title{
Re: X-Linked TEX11 Mutations, Meiotic Arrest and Azoospermia in Infertile Men
}

\author{
Alexander N. Yatsenko1, Andrew Georgiadis1, Albercht Röpke2, Andrea J. Berman2, \\ Thomas Jaffe1, Marta Olszewska4, Birgit Westernströer3, Joseph Sanfilippo1, Maciej Kurpisz4, \\ Aleksandar Rajkovic1, Svetlana A. Yatsenko1, Sabine Kliesch3, Stefan Schlatt3, Frank Tüttelmann2 \\ 1 University of Pittsburgh School of Medicine, Departments of Obstetrics, Gynecology and Reproductive Sciences and Urology, Pittsburgh, Pennsy/vania \\ 2University of Pittsburgh, Kenneth P. Dietrich School of Arts and Sciences, Department of Biological Sciences, - all in Pittsburgh; the Institute of \\ Human Genetics, Pittsburgh, Pennsylvania \\ 3University of Münster, Center of Reproductive Medicine and Andrology, Münster, Germany \\ 4 Polish Academy of Sciences, Department of Reproductive Biology and Stem Cells, Institute of Human Genetics, Poznan, Polish
}

New England Journal of Medicine 2015;372:2097-2107.

\section{EDITORIAL COMMENT}

Infertility affects nearly 10\% of couples and about half of these suffer from male infertility. The most severe form of male infertility is nonobstructive azoospermia (NOA) which occurs mostly due to failure in sperm production in the testes. Current genetic tests reveal around 10\% of diagnosis in all patients with azoospermia such as 47,XXY (Klinefelter syndrome) or Y chromosome microdeletions. However, most patients with NOA remain idiopathic, which is very frustrating for them. In this study, the authors performed array comparative genomic hybridization (aCGH) in blood samples obtained from men with NOA and mutation analysis using Sanger sequencing expressed 11 (TEX11) gene in NOA patients and controls. Overall mutations were found in 7 of 289 men with NOA (2.4\%) and were absent in 384 men with normal sperm concentration. 5 of those TEX11 mutations were detected in 33 azoospermic patients (15\%) who had a diagnosis of maturation arrest in pathological assessment. Immunohistochemical analysis showed specific cytoplasmic TEX11 expression in late spermatocytes like round and elongated spermatids. However, in maturation arrest pathology with TEX11 mutations, no TEX11 expression was noted. This study showed that X chromosome-linked TEX 11 gene mutations is important for the diagnosis of men with NOA, especially with meiotic arrest.

Recently, two new studies were published regarding new discoveries of genes affecting testicular sperm production in azoospermic brothers in two different Turkish families. Okutman et al. (1) showed that homozygous mutation TEX15 in three azoospermic brothers was the cause of a spermatogenic defect that is transmitted according to an autosomal recessive trait in a consanguineous Turkish family. All azoospermic brothers had hypoplasic testis volumes (10-12 ml) and meiotic arrest in pathological assessment. All azoospermic men had sperm recovery by Micro TESE operation. Another study by Ramasamy et al. (2) showed a novel mutation in the neuronal PAS2 domain (NPAS2) in a consanguineous Turkish family. Again, the family segregation of the mutation showed the presence of homozygous mutation in three NOA brothers and a heterozygous mutation in the mother. The mutation was not found in a cohort of 500 Turkish patients and in matching 50 fertile controls. These azoospermic brothers had hypoplasic testis volume (12-14 ml), high FSH and normal testosterone levels. Two of the brother had micro TESE operation; one of them with success of sperm recovery but the other had a negative result. In conclusion, these recent studies showed that using new genetic analysis, such as next-generation sequencing, whole-exome sequencing would be a powerful tool for identifying idiopathic male infertility and men with NOA. New studies will shed light in understanding the pathophysiology and treatment of male infertility.

\section{REFERENCES}

1. Okutman O, Muller J, Baert Y, Serdarogullari M, Gultomruk M, Piton A, Rombaut C, Benkhalifa M, Teletin M, Skory V, Bakircioglu E, Goossens E, Bahceci M, Viville S. Exome sequencing reveals a nonsense mutation in Tex15 causing spermatogenic failure in a Turkish family. Hum Mol Genet 2015;21. pii: ddv290. [Epub ahead of print]

2. Ramasamy R, Bakırcıoğlu ME, Cengiz C, Karaca E, Scovell J, Jhangiani SN, Akdemir ZC, Bainbridge M, Yu Y, Huff C, Gibbs RA, Lupski JR, Lamb DJ. Whole-exome sequencing identifies novel homozygous mutation in NPAS2 in family with nonobstructive azoospermia. Fertil Steril 2015;104:286-291. 